\title{
SOFTWARE MOVIL PARA RECONOCIMIENTO, SEGUIMIENTO Y CONTROL DE LAS PLANTAS DEL VIVERO INTELIGENTE DE LA UNIVERSIDAD FRANCISCO DE PAULA SANTANDER OCAÑA

\author{
DEVELOPMENT OF MOBILE SOFTWARE FOR RECOGNITION, \\ MONITORING AND CONTROL OF PLANTS OF THE INTELLIGENT NURSE \\ OF THE UNIVERSITY FRANCISCO DE PAULA SANTANDER OCAÑA
}

\author{
MSc. Luis Anderson Coronel-Rojas, MSc. Dewar Rico-Bautista, MSc. Fabián \\ Ranulfo Cuesta-Quintero, MSc. Edwin Barrientos-Avendaño, Est. Eimar Alveiro \\ Pedraza Villadiego*
}

* Universidad Francisco de Paula Santander Ocaña. Grupo de Ingeniería en Innovación, Tecnología y Emprendimiento GRIITEM. Vía Acolsure, Sede el Algodonal, Ocaña Norte de Santander, Colombia. 5690088

E-mail: \{ebarrientosa, dwricob, fcuestaq, lacoronelr, eapedrazav $\} @$ ufpso.edu.co.

Resumen: Este artículo tiene como objetivo mostrar los aspectos del desarrollo de una aplicación móvil. Permite dar soporte a los procesos de registro de hoja de vida de las diferentes plantas objeto de estudio del jardín Botánico Jorge Enrique Quintero Arenas de la universidad Francisco de Paula Santander Ocaña. En la primera fase, se realizó un análisis de los diferentes formatos físicos que actualmente lleva el administrador de la granja para la toma de datos de interés. En la segunda, la sistematización de la captura de información a través de interfaces amigables fácil de utilizar sobre una App Móvil con características que permitirán identificar a cada planta con tecnología códigos QR. Se agilizaron las consultas, reportes, trazabilidad e identificación de procedimientos e intervenciones realizadas a cada una de las plantas que están en proceso de supervisión por motivos científicos e investigativos, logrando ser una herramienta de apoyo para la toma de decisiones.

Palabras clave: Smart, Códigos QR, APP, Jardín botánico, Sistema, Tecnología.

\begin{abstract}
This article aims to show the aspects of the development of a mobile application. It allows to support the processes of registration of the curriculum of the different plants under study of the Botanical Garden Jorge Enrique Quintero Arenas of the Francisco de Paula University Santander Ocaña. In the first phase, an analysis was made of the different physical formats that the farm administrator currently takes for the collection of data of interest. In the second, the systematization of the capture of information through easy-to-use friendly interfaces on a Mobile App with features that will allow identifying each plant with QR codes technology. Consultations, reports, traceability and identification of procedures and interventions made to each of the plants that are in the process of supervision for scientific and investigative reasons were streamlined, making it a support tool for decision making.
\end{abstract}

Keywords: Smart, QR codes, APP, Botanical garden, System, Technology. 


\section{INTRODUCCIÓN}

La constante incursión de aplicativos móviles en el quehacer de las personas(Quiroga-Parra, TorrentSellens, \& Murcia-Zorrilla, 2017) y el uso constante de información gracias a la facilidad que dichas aplicaciones generan(Beltrán-Raml'lirez, Maciel-Arellano, Gómez-Barba, Stokes, \& Gonzalez-Sandoval, 2015), se han venido mostrando como algunos de los factores determinantes y explicativos del incremento del a eficiencia, la productividad de los trabajadores(Quiroga-Parra et al., 2017) ya que el tener la información disponible cuando se necesita es un fundamental para la toma de decisiones(González \& Soler, 2017) (JEG Plaza, MAR Nuñez. 2017)

Los procesos llevados a cabo en el jardín botánico Jorge Enrique Quintero Arenas se han venido llevando desde su creación a través de formatos impresos que dificultan la administración e investigaciones que se realizan en el mismo, como la toma de datos de plantas objeto de estudio, trazabilidad de intervenciones realizadas en diferentes áreas del jardín y demás tareas de administración, por tal razón se vio la necesidad de crear una aplicación móvil que permita mejorar la toma de información bajo una identificación de cada planta soportados por cogidos QR, el cual permitirá una eficiencia en la toma de decisiones por parte del administrador del jardín botánico Jorge Enrique Arenas Quintero. Dicha aplicación se encuentra enmarca en el proyecto de jardín botánico inteligente que se encuentra en desarrollo, por lo que se hizo necesario realizar un estado del arte que permitiera focalizar como son administrados los jardines botánicos en diferentes partes del mundo (Rico-Bautista, Parra-Valencia, \& Guerrero, 2017).

\section{ESTADO DEL ARTE}

Se analizaron artículos relacionados sobre jardines botánico inteligente que permitieron contextualizar la administración, revisando los aportes que genera la tecnología en el control de actividades del día a día en el jardín botánico.

Hoy en día, los jardines botánicos se consideran los espacios verdes públicos más importantes e influyentes en la mayoría de los países del mundo. Soportan aplicaciones científicas y de investigación en los campos de la botánica, la jardinería, la conservación y la ecología vegetal (Barkoosaraei \& Moshiri, 2017). Varían ampliamente en diseño y propósito, pero la mayoría están típicamente asociados con la conservación del medio ambiente, la educación o la interpretación histórica. Sin embargo, los estudios han demostrado que los visitantes del jardín botánico a menudo están motivados por intereses recreativos y de ocio (Wassenberg, Goldenberg, \& Soule, 2015).

Una comunidad puede volverse inteligente y ecológica a través del despliegue estratégico de la infraestructura y los servicios de Tecnología de la Información y las Comunicaciones para alcanzar los objetivos de la política de sostenibilidad (Luvisi \& Lorenzini, 2014). Uno de los principales atributos que distingue a un jardín botánico de un jardín puramente expositivo es la práctica de acceder a las plantas cuando ingresan a la colección y luego llevar registros de este material a lo largo de su vida (Walter, 1991), (Agostini et al., 2017). Sin embargo, las plantas no sólo constituyen un espacio verde útil para contrastar los efectos de la contaminación urbana o proporcionar beneficios eco sistémicos a los residentes, sino que también pueden utilizarse como bioindicadores y su participación en las redes de comunicación puede representar una contribución significativa para construir una comunidad inteligente y verde (Barkoosaraei \& Moshiri, 2017) (CJ Torres, JF Archila, ML Tronco. 2013)

La infraestructura de sensores para el jardín reúne y analizan datos sobre cambios de clima y condiciones de humedad del suelo y luego se conecta al teléfono del usuario con alertas oportunas. Monitorea continuamente condiciones y alerta al usuario de los cambios que requieren acción inmediata. A diferencia de los aspersores preestablecidos, un buen sistema podrá controlar automáticamente el sistema de riego existente basado en los datos recopilados por el sensor de jardín (Biswal, Singh, Jeberson, \& Dhar, 2015). Los parámetros se almacenan en estación de trabajo local y se comparte con la nube para ser mostrado en un sistema web (Santos \& RicoBautista, 2009), el objetivo principal del diseño es resolver la gestión de problemas, mantenimiento, detección de fallas y tiempo medio de reparación (Basuvaiyan \& Rathinasabapathy, 2017). Siendo coherentes la infraestructura debe estar soportada por energías solares. (JA Villabona, CL Sandoval. 2018)

Las universidades tienen un papel que desempeñar para abordar temas en relación con la disponibilidad de un personal debidamente cualificado, a través de la provisión de graduados 
en áreas de conocimiento soporte de la agricultura. Los jardineros humanos con experiencia son muy buenos para tratar las plantas de forma individual. Desafortunadamente, contratar a un equipo de jardineros para llevar a cabo esta tarea en grandes invernaderos no es rentable(Verma, Lamb, Reid, \& Wilson, 2016).

\section{METODOLOGIA}

\subsection{Tipo de investigación}

Según Tamayo (2004), "La investigación descriptiva trabaja sobre realidades de hechos, y su característica fundamental es la de presentarnos una interpretación correcta" (p. 46). Es por ello que en este proyecto se ha definido utilizar el tipo de investigación descriptiva con el fin de analizar los diferentes formatos físicos utilizados para la administración del jardín botánico con el obj etivo de plantear las interfaces gráficas y las funcionalidades de la aplicación móvil.

\subsection{Población}

La población objeto de estudio involucrada está conformada por el administrador del jardín botánico Jorge Enrique Quintero Arenas

\subsection{Muestra}

Según Tamayo (2004), "La muestra es parte representativa de una población" (p. 173). Pero al ser la población muy limitada se tomará el $100 \%$; dicha población estará conformada por el administrador del Jardín botánico.

\section{RESULTADOS}

En el proceso de desarrollo de la App se analizó los diferentes formatos que se llevan de forma manual por parte del administrador del jardín botánico con el objetivo de identificar las características relevantes de cada una de las plantas de objeto de estudio por los investigadores.

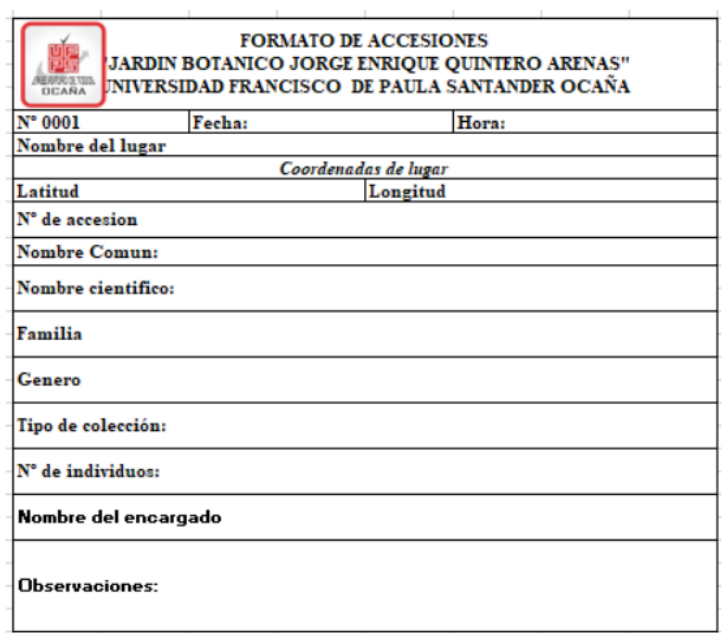

Fig. 1. Formato de Accesiones.

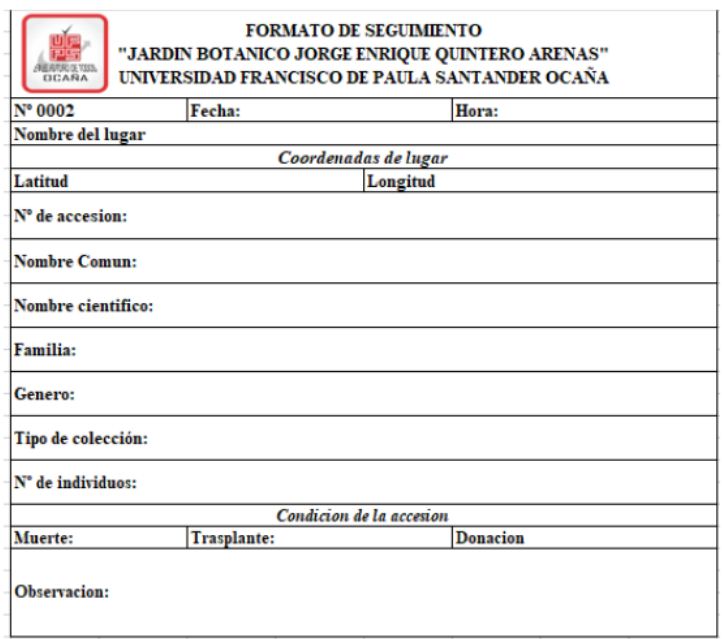

Fig. 2. Formato de Accesiones.

Una vez se tiene el análisis de procede a la elaboración de la aplicación móvil logrando desarrollar una aplicación con características de usabilidad y ergonomía bastante simple de manejar que permitiera satisfacer las necesidades de los investigadores

La aplicación comienza con la siguiente interfaz 


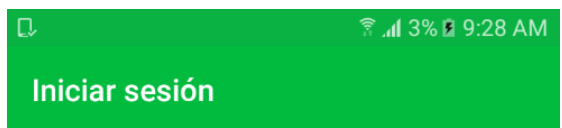

Email

Contraseña

¿Has olvidado tu contraseña?

INICIAR SESIÓN

Fig. 3. Inicio de sesión.

El usuario se debe estar registrado para poder adquirir las crede nciales necesarias para ingresar al sistema, una vez se autentica se le da acceso al menú de opciones donde podrá realizar funcionalidades entre las que se encuentran, registrar una planta, evaluar características detectadas por los investigadores y realizar consultas inteligentes con el objetivo de tomar decisiones en base a información de interés.

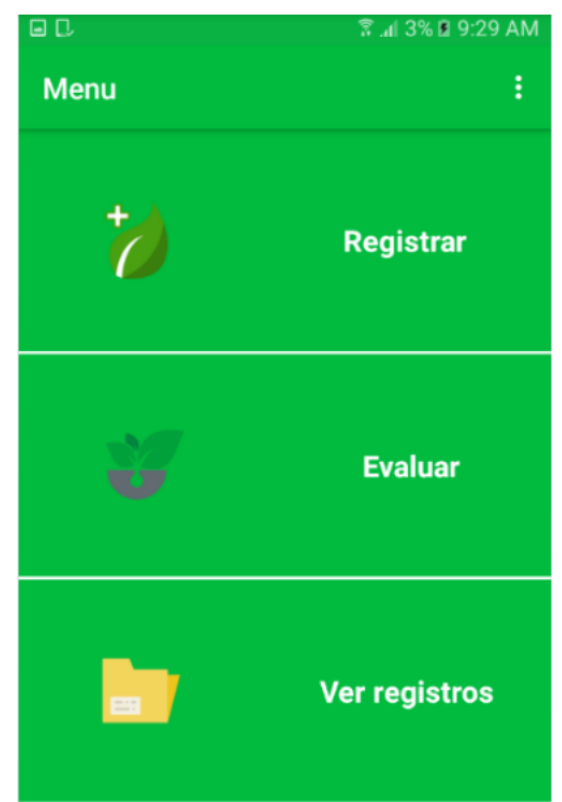

Fig. 4. Menú de opciones.

En la interfaz de registro de plantas los investigadores se encontraran con un formulario donde se podrá capturar información de las plantas objeto de estudio presente en los viveros del jardín botánico

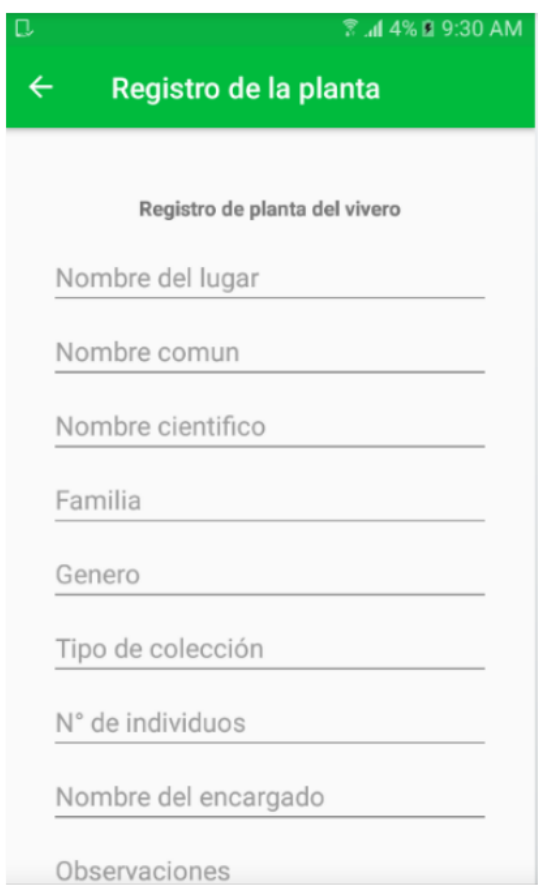

Fig. 5. Registro de planta.

Dentro del registro de cada planta se podrá identificar cada una de ellas con un cogido QR que nos permitirá agilizar el proceso de búsqueda y trazabilidad de las diferentes intervenciones que se estén realizando objeto de investigaciones como control de plagas, formas, crecimiento, etc.

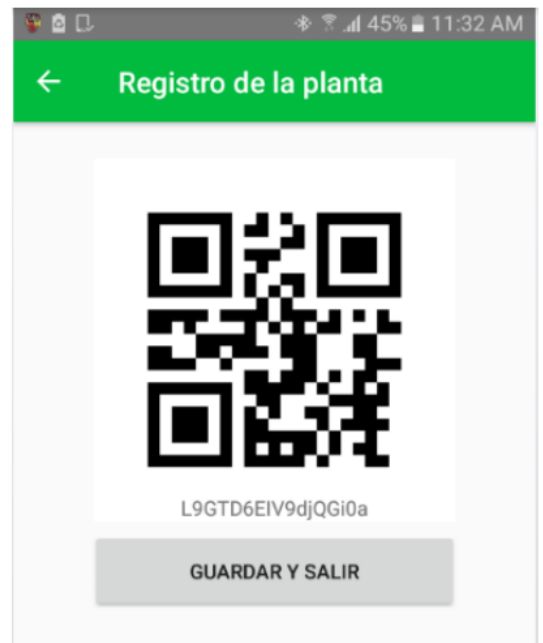

Fig. 6. Identificación código $Q R$.

Una vez las plantas están identificadas en su ubicación y características, el administrador de la granja inteligente podrá de forma muy rápida realizar trabajos del día a día como control de 
plagas, seguimientos a muertes, trasplantes, donaciones que se pueden presentar.

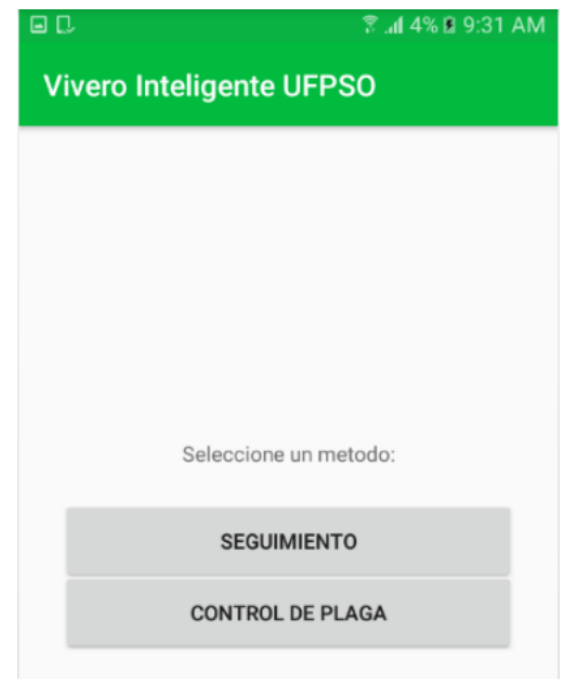

Fig. 7. Opciones.

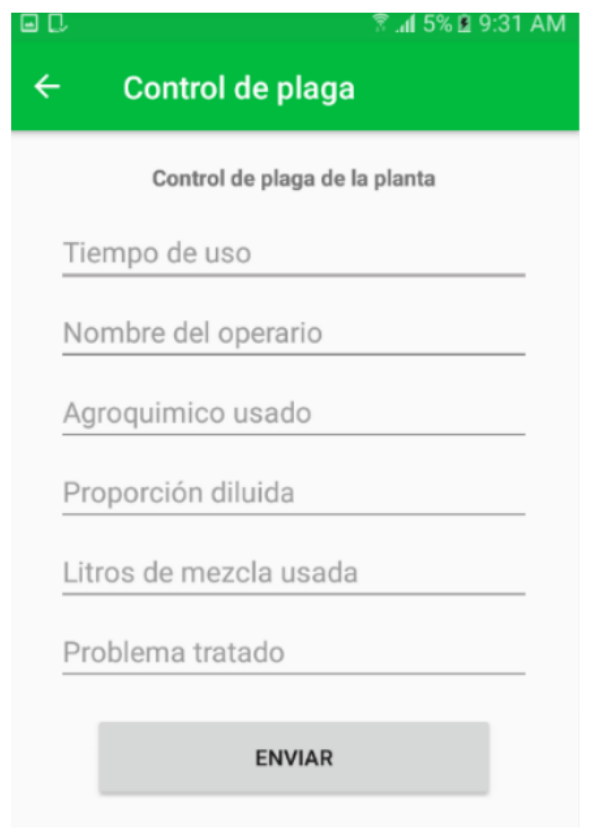

Fig. 8. Control de plagas.

Todas estas interfaces permitirán al administrador e investigadores del jardín botánico inteligente obtener información en tiempo real bajo reportes de interés para la toma de decisiones.

\section{CONCLUSIONES}

Este artículo permitió dar a conocer una las múltiples aplicaciones que actualmente se están elaborando encaminados en fortalecer el jardín botánico inteligente que actualmente se encuentra en desarrollo dentro de la universidad Francisco de Paula Santander.

Dicha aplicación es una herramienta que permitirá dar soporte de forma rápida y ágil a los diferentes procesos que actualmente se llevan a cabo dentro del jardín botánico, tanto a nivel investigativo como de administración del mismo.

La implementación del código QR para identificar cada planta objeto de estudio es un plus que permitirá dar soporte a otros proyectos en curso como lo es el software de realidad aumentada para el recorrido del jardín Botánico por parte de visitantes ya que los QR servirán de marcadores para dar a conocer las características de plantas nativas como orquídeas, heliconias, árboles que embellecen el ambiente natural (Lobo Jaime \& Rico-Bautista, 2015).

La toma de decisiones en tiempo real es fundamental para el jardín botánico, por tal razón dicha aplicación será de gran ayuda por la facilidad de operación de administradores e investigadores.

\section{AGRADECIMIENTOS}

Para el desarrollo de esta investigación fue fundamental la ayuda prestada por el administrador del jardín botánico quien estuvo siempre dispuesto a colaborar para el buen desarrollo de la aplicación.

\section{BIBLIOGRAFIA}

Agostini, A., Alenyà, G., Fischbach, A., Scharr, H., Wörgötter, F., \& Torras, C. (2017). A cognitive architecture for automatic gardening. Computers and Electronics in Agriculture, 138, 69-79. https://doi.org/10.1016/j.compag.2017.04.01 5

Barkoosaraei, M. H. D. A., \& Moshiri, S. (2017). Designing of Genow Botanical Garden with Sustainable Architecture Approach. TARIH KULTUR VE SANAT ARASTIRMALARI DERGISI-JOURNAL OF HISTORY CULTURE AND ART RESEARCH, 6(4), 1211-1226. https://doi.org/10.7596/taksad.v6i4.1140

Basuvaiyan, S., \& Rathinasabapathy, V. (2017). IoT based Solar Photo Voltaic Monitoring System.

Beltrán-Raml’lirez, R., Maciel-Arellano, R., Gómez-Barba, L., Stokes, L., \& GonzalezSandoval, C. (2015). Mobile applications utilized for the prevention of potential 
epidemics in smart cities. In Smart Cities Conference (ISC2), 2015 IEEE First International (pp. 1-4).

Biswal, E. V., Singh, E. H. M., Jeberson, W., \& Dhar, E. A. S. (2015). Greeves: A Smart Houseplant Watering and Monitoring System, 4(7), 2499-2507.

C. J. Torres, J. F. Archila, M. L. Tronco, M. Becker, A. J. V. Porto, A. J. Tiberti. (2013). Estudio cinemático de una plataforma robótica para agricultura. REVISTA COLOMBIANA DE TECNOLOGÍAS DE AVANZADA, ISSN: 1692-7257. 2(22).

Torres, C., Archila, J., Tronco, M., Becker, M., Viera Porto, A., \& Tiberti, A. (2013). Estudio cinemático de una plataforma robótica para agricultura. REVISTA COLOMBIANA DE TECNOLOGÍAS DE AVANZADA, 2(22).

González, M. Á., \& Soler, M. O. (2017). El proceso de toma de decisiones profesionales a través del coaching. Electronic Journal of Research in Education Psychology, 7(18).

JA Villabona, CL Sandoval, (2018), Tecnificación Para El Control De Presión Y Emulación De Riego Usando El Control Lógico Programable Y Software Totally. Revista Colombiana de Tecnologías de Avanzada ISSN: 1692-7257.

JEG Plaza, MAR Nuñez. (2017), Formación en competencias específicas para la industria del software colombiano. Experiencias del uso del aprendizaje basado en proyectos Revista Colombiana de Tecnologías de Avanzada ISSN: $1692-7257$.

Lobo Jaime, C. E., \& Rico-Bautista, D. (2015). Reconocimiento de patrones: Fase de pruebas en software de acceso a información institucional basado en realidad aumentada. Puente Revista Científica, 9(1), 39-44. Retrieved from https://revistas.upb.edu.co/index.php/puente/ article/view/7120

Luvisi, A., \& Lorenzini, G. (2014). RFID-plants in the smart city: Applications and outlook for urban green management. Urban Forestry and Urban Greening, 13(4), 630-637. https://doi.org/10.1016/j.ufug.2014.07.003

Quiroga-Parra, D. J., Torrent-Sellens, J., \& MurciaZorrilla, C. P. (2017). Las tecnolog $\{\backslash \backslash \mid i\}$ as de la información en América Latina, su incidencia en la productividad: Un análisis comparado con pa $\{\backslash \backslash \mathrm{i}\}$ ses desarrollados. Dyna, 84(200), 281-290.

Rico-Bautista, D., Parra-Valencia, J. A., \& Guerrero, C. D. (2017). IOT: UNA
Revista Colombiana de Tecnologías de Avanzada

APROXIMACION DESDE CIUDAD INTELIGENTE A UNIVERSIDAD INTELIGENTE. Revista Ingenio UFPSO, 13(1), 9-20.

Santos, L. M., \& Rico-Bautista, D. (2009). SERVICIOS WEB EN TELEFONÍA CELULAR Web Services in Cellular Telephony, 15(42), 363-368. https://doi.org/DOI: http://dx.doi.org/10.22517/23447214.2635

Verma, N. K., Lamb, D. W., Reid, N., \& Wilson, B. (2016). Comparison of Canopy Volume Measurements of Scattered Eucalypt Farm Trees Derived from High Spatial Resolution Imagery and LiDAR. REMOTE SENSING, 8(5). https://doi.org/10.3390/rs8050388

Walter, K. S. (1991). Computerized plant record systems for botanic gardens. Tropical Botanic Gardens. ACADEMIC PRESS LIMITED. https://doi.org/10.1016/B978-012-346850-5.50035-1

Wassenberg, C. L., Goldenberg, M. A., \& Soule, K. E. (2015). Benefits of botanical garden visitation: A means-end study. Urban Forestry and Urban Greening, 14(1), 148155. https://doi.org/10.1016/j.ufug.2015.01.002

Tamayo, M. (2004). El proceso de la investigación científica. Editorial Limusa. 